\title{
Caveolin-1 regulates the expression of tight junction proteins during hyperoxia-induced pulmonary epithelial barrier breakdown
}

\author{
Shuyan Xu, Xindong Xue, Kai You and Jianhua Fu*
}

\begin{abstract}
Background: Bronchopulmonary dysplasia (BPD) is a common complication in preterm infants that involves the downregulation of tight junction (TJ) proteins. However, the mechanism underlying downregulation of the expression of TJ proteins during at the early stages of hyperoxia-induced BPD remains to be understood. Here, we aimed to identify the role of caveolin-1 (Cav-1) in hyperoxia-induced pulmonary epithelial barrier breakdown.

Methods: First, we established an in vitro pulmonary epithelial barrier models using primary type II alveolar epithelial cells (AEC-II) from newborn rats. AEC-II was assigned to the hyperoxic $\left(85 \% \mathrm{O}_{2} / 5 \% \mathrm{CO}_{2}\right)$ or normoxic $\left(21 \% \mathrm{O}_{2} / 5 \%\right.$ $\mathrm{CO}_{2}$ ) groups. Second, AEC-II was transfected with Cav-1-siRNA to downregulate Cav-1 under normoxic exposure. Third, AEC-II was transfected with a cDNA encoding Cav-1 to upregulate Cav-1 expression under hyperoxic exposure. Then, expression levels of Cav-1 and TJ proteins were examined by immunofluorescence staining, reverse transcriptionpolymerase chain reaction, and Western blotting. The TJ structures visualized using a transmission electron microscope, and transepithelial resistance and apparent permeability coefficient of fluorescein isothiocyanate-dextran, which are indicators of barrier function, were measured.
\end{abstract}

Results: Our data showed that exposure to hyperoxia disrupted the structure and function of the pulmonary epithelial barrier and decreased the ZO-1, occludin, claudin-4, and Cav-1 expression levels. Moreover, Cav-1 knockdown attenuated the expression of the other three genes and disrupted pulmonary epithelial barrier structure and function under normoxic exposure. However, Cav-1 upregulation markedly antagonized the hyperoxia-induced pulmonary epithelial barrier destruction and TJ protein loss.

Conclusions: This is the first study to present evidence illustrating the novel role of Cav-1 downregulation-mediated TJ protein loss in pulmonary epithelial barrier destruction during BPD.

Keywords: Caveolin-1, Tight junction protein, Pulmonary epithelial barrier, Hyperoxia, Bronchopulmonary dysplasia

\section{Background}

Bronchopulmonary dysplasia (BPD) is one of the most severe complications in preterm infants, which is a lung injury that occurs due to high oxygen exposure and mechanical ventilation [1]. Apart from hyperoxia and mechanical ventilation, infection and preterm birth are also involved in causing BPD [2]. Although the occurrence of severe BPD has been reduced due to advances in clinical treatment, improvements in mechanical ventilation, and the clinical application of lung surfactant and

\footnotetext{
* Correspondence: fujh@sj-hospital.org

Department of Pediatrics, Shengjing Hospital of China Medical University, Shenyang 110004, Liaoning, China
}

glucocorticoids in preterm infants, the incidence remains to be high in very low birth weight infants [3]. Infants with BPD require long-term oxygen supplementation after hospitalization, as BPD is associated with potential multiple complications in the respiratory and nervous system $[4,5]$. Therefore, there is an urgent need to understand the cause of BPD and use this information to develop prevention and treatment strategies.

The mechanism of BPD remains to be fully understood; however, an increasing body of evidence indicates that the main pathological changes include early-stage pulmonary edema [6-8] and late-stage defects in alveolar and microvasculature development [9]. Current research mainly 
focuses on these defects, but the mechanism of pulmonary edema in BPD has not been studied in detail. In our previous study, we used a hyperoxia-induced BPD model and showed that BPD is accompanied by destruction of TJ structures in the lung, increased permeability of the pulmonary epithelial barrier, and a decline in the concentrations of certain TJ proteins [7]. Thus, it is quite possible that the pulmonary edema at an early stage of BPD resulted from the destruction of structure and function of pulmonary epithelial barrier caused by the loss of $\mathrm{TJ}$ proteins.

TJ is a protein complex among neighboring cells, which includes integral proteins such as occludin, claudins, and tricellulin, and adaptor proteins like cingulin and ZOs [10]. Previous research has shown that ZO-1 may serve as a link between the TJ proteins such as occluding and the actin cytoskeleton [11]. ZO-1, occludin, and claudin- 4 are important components of $\mathrm{TJ}$ in pulmonary epithelial barrier [12]. Occludin is a type of transmembrane protein and is required for maintaining the integrity of lung epithelial barrier [13, 14]. ZO-1 is a scaffold protein that promotes the assembly of proteinprotein complex and influences the structure and function of lung epithelial barrier $[15,16]$. Claudin- 4 is also a type of transmembrane protein that improves the barrier function of pulmonary epithelial barrier by promoting pulmonary fluid-clearance function $[17,18]$. In our previous study, in the hyperoxia-induced BPD model, TJ protein ZO-1 and occludin were both found to decrease, and it was therefore predicted that this may be the mechanism underlying of hyperoxia-induced destruction of lung epithelial barrier [7]. Therefore, we aimed to analyze the expression of ZO-1, occludin, and claudin-4 in relation to caveolin-1 in this study.

Tight junction (TJ) protein expression and assembly are regulated by several factors such as tumor necrosis factor- $\alpha$ (TNF- $\alpha$ ), interferon- $\gamma$ (IFN- $\gamma$ ) [19], matrix metalloproteinases (MMPs) [20], microRNAs [21], small GTPases [22], and phosphorylation-related regulation [23]. Caveolin-1 (Cav-1) is an important structural and regulatory component of caveolae, which is involved in multiple physiological processes including vesicle transportation and cellular signal transduction [24, 25]. Cav-1 has also been indicated to be involved in regulating the assembly of TJ proteins [26] and further influencing the function of blood-brain barrier [27] and intestinal epithelial barrier [28]. In the lung, Cav-1 is highly expressed in epithelial cells, fibroblast, vascular endothelial cells, and inflammatory cells [29]. Cav-1 downregulation in lung tissue has been shown to be highly correlated with BPD [30]. Gao et al. reported that pulmonary injection of Cav-1-siRNA could increase the permeability of the pulmonary epithelial barrier in rats [31], but the underlying mechanism remains to be elucidated. Therefore, we used an in vitro pulmonary epithelial barrier model to investigate the role of Cav-1 in hyperoxia-induced destruction of the pulmonary epithelial barrier, and to elucidate the molecular mechanisms underlying pulmonary epithelial barrier destruction in BPD. The results of this study provide a theoretical basis for preventing and treating pulmonary edema at an early stage of BPD.

\section{Methods}

\section{Isolation of type II alveolar epithelial cells (AEC-II)}

Pregnant Wistar rats weighing 200-220 g were purchased from the Experimental Animal Center of China Medical University. Each pregnant Wistar rat was independently feeding, and gave birth to litters via natural birth at 22 days of pregnancy. AEC-II was isolated as described previously [6]. Within $12 \mathrm{~h}$ of birth, newborn Wistar rats were anesthetized by intraperitoneal injection of $10 \%$ chloral hydrate $(3 \mathrm{~mL} / \mathrm{kg})$. After cardiopulmonary lavage, the lung tissues were dissected and sectioned into $1-\mathrm{mm}^{3}$ pieces in a sterile condition. The samples were digested in $0.25 \%$ trypsin EDTA (Gibco Life Technologies, Grand Island, NY, USA) followed by filtration, and further digested with $0.1 \%$ collagenase I (Gibco Life Technologies). The cells were harvested by centrifugation at $800 \mathrm{rpm}$, resuspended and incubated in a $5 \% \mathrm{CO}_{2}$ incubator at $37{ }^{\circ} \mathrm{C}$. To remove fibroblasts, the medium was poured every $1 \mathrm{~h}$ for three times. To remove macrophages, nonadherent cells were transferred to rat immunoglobulin G (IgG)-coated plates and incubated for $1 \mathrm{~h}$. The viability ( $>95 \%)$ and purity ( $>90 \%$ ) of AEC-II were tested by trypan blue staining and immunostaining of AEC-II-specific marker surfactant protein $\mathrm{C}(\mathrm{SP}-\mathrm{C})$, respectively.

All procedures and animal experiments were approved by the Animal Care and Use Committee of China Medical University.

\section{Establishment of an in vitro pulmonary epithelial barrier model}

Based on previous protocol for establishing an in vitro pulmonary epithelial barrier model [32, 33], AEC-II was seeded onto Transwell Clear inserts (diameter, $6.5 \mathrm{~mm}$; pore size, $0.4 \mu \mathrm{m}$; Corning Costar, Cambridge, MA, USA) at a density of $2-3 \times 10^{6}$ cells $/ \mathrm{mL}$ and cultured in Dulbecco's modified Eagle's medium (DMEM)/F12 (Hyclone, Logan, UT, USA) with $10 \%$ fetal bovine serum (FBS; Clark, Seabrook, MD, USA). The medium was changed after $24 \mathrm{~h}$. The pulmonary epithelial barrier could be successfully established after consecutive in vitro culture for 7 days and then randomly grouped into hyperoxic and normoxic groups. The hyperoxic group was incubated in an $85 \% \mathrm{O}_{2} / 5 \% \mathrm{CO}_{2}$ incubator (CB150, Binder, Tuttlingen, Germany), while the normoxic group was incubated in a $21 \% \mathrm{O}_{2} / 5 \quad \% \mathrm{CO}_{2}$ 
incubator (3111, Thermo Fisher Scientific, Marietta, OH, USA). Cells were collected for RT-PCR, Western blot and immunofluorescence staining, and simultaneously, pulmonary epithelial barrier function was measured after $0,24,48$, and $72 \mathrm{~h}$ under exposure to hyperoxic or normoxic conditions.

\section{Transfection of primary cultured AEC-II}

Primary cultured AEC-II were seeded onto Transwell Clear inserts and cultured to 60-70\% confluence before transfection. For Cav-1-siRNA transfection, cells were transfected with $10 \mathrm{~nm}$ of a rat Cav-1-specific siRNA (5'-GGCAUAGCACAAGUAAUAGUCUGTA-3'; Cat.\# SR500200; OriGene Technologies, Rockville, MD, USA) or $10 \mathrm{~nm}$ of a non-silencing scrambled control siRNA (Cat.\#SR30004;OriGene Technologies) using a siTran 1.0 transfection reagent (OriGene Technologies), according to the manufacturer's protocol. For Cav-1-cDNA transfection, cells were exposed to hyperoxia $\left(85 \% \mathrm{O}_{2}\right.$ and $5 \% \mathrm{CO}_{2}$ ) and transfected with Cav-1 cDNA plasmid (1 $\mu \mathrm{g} / \mathrm{mL}$; Cat.\#RR200414; OriGene Technologies) or with an empty vector plasmid $(1 \mu \mathrm{g} / \mathrm{mL}$; Cat.\#PS100001; OriGene Technologies) as control using Turbofectin 8.0 transfection reagent (OriGene Technologies), following the manufacturer's instructions.

Cav-1 mRNA and protein expression levels were monitored using reverse transcription-polymerase chain reaction (RT-PCR) and Western blot analysis, respectively, to determine the best concentration of Cav-1siRNA or Cav-1 cDNA plasmid for transfection and the best post-transfection time for the lowest and highest Cav-1 expression. Cells transfected with siRNA or cDNA plasmid were harvested at 48 and $72 \mathrm{~h}$ after transfection, respectively, before further experiments.

\section{RT-PCR}

The total RNA was extracted from pulmonary epithelial cells using Trizol reagent (Invitrogen, Camarillo, CA, USA), and the purity was determined by spectrophotometer $\left(\mathrm{OD}_{260} / \mathrm{OD}_{280}\right)$. The total RNA $(1 \mu \mathrm{g})$ was reversetranscribed to cDNA using SuperScript III First-Strand Synthesis System (Invitrogen). Primers were designed and synthesized by TaKaRa Inc. (Shiga, Japan) as follows: Cav1, forward: 5'-CGGGAACAGGGCAACATCTAC-3', reverse: 5'-CTTCTGGTTCCGCAATCACATC-3'; ZO-1, forward: 5'-CCATCTTTGGACCGATTGCTG-3', reverse: 5'-TAATGCCCGAGCTCCGATG-3'; Occludin, forward: 5'-GTCTTGGGAGCCTTGACATCTTG-3', reverse: 5'GCATTGGTCGAACGTGCATC-3'; Claudin-4, forward: 5'-ACGAGACCGTCAAGGCCAAG-3', reverse: 5'-GTCC AGGACACAGGCACCATAA-3'; $\beta$-actin, forward: 5'-GG AGATTACTGCCCTGGCTCCTA-3', reverse: 5'-GACTC ATCGTACTCCTGCTTGCTG-3'. The conditions of amplification were as follows: 30 cycles at $95{ }^{\circ} \mathrm{C}$ for $30 \mathrm{~s}$,
$55{ }^{\circ} \mathrm{C}$ for $40 \mathrm{~s}$, and $75{ }^{\circ} \mathrm{C}$ for $1 \mathrm{~min}$ in a thermal cycler (ABI, Vernon, CA, USA). The PCR amplicons were densitometrically analyzed by $2.5 \%$ agarose gel electrophoresis using ImageJ software (National Institutes of Health, Bethesda, MD, USA). The relative expression level of target genes was calculated after normalization to the levels of $\beta$-actin.

\section{Western blot analysis}

Briefly, equal amounts of protein were extracted from pulmonary epithelial cells and separated by electrophoresis on $10 \%$ sodium dodecyl sulfate (SDS) polyacrylamide gel and transferred to polyvinylidene difluoride (PVDF) membranes (Millipore, Billerica, MA). After blocking with $5 \%$ bovine serum albumin (BSA), the PVDF membranes were incubated with the following primary antibodies at $4{ }^{\circ} \mathrm{C}$ overnight: rabbit anti-rat polyclonal antibody against Cav-1 (1:1000, Cat.\#3238, Cell Signaling, Danvers, MA), rabbit antirat polyclonal antibody against ZO-1 (1:500, Cat.\#402200, Invitrogen, San Francisco, CA, USA), rabbit anti-rat polyclonal antibody against occludin (1:500, Cat.\#71-1500, Invitrogen), mouse anti-rat monoclonal antibody against claudin-4 (1:500, Cat.\#32-9400, Invitrogen), and rabbit anti-rat polyclonal antibody against $\beta$-actin (1:1000, Cat. sc-130657, Santa Cruz Biotechnology, CA, USA). After washing, the membranes were incubated with horseradish peroxidaseconjugated secondary antibody (Cell Signaling, Beverly, MA, USA). The membranes were washed again and visualized with chemiluminescence substrate (ECL kit; Santa Cruz Biotechnology). The optical density of the protein bands was analyzed using Image J software and normalized to that of $\beta$-actin.

\section{Immunofluorescence staining}

Pulmonary epithelial cells were fixed in $4 \%$ paraformaldehyde for $30 \mathrm{~min}$ and washed thrice with phosphatebuffered saline (PBS). After blocking with $10 \%$ goat serum, cell monolayers were incubated with the following primary antibodies: rabbit anti-rat polyclonal antibody againstZO-1 (1:100), rabbit anti-rat polyclonal antibody against occludin (1:100), and mouse anti-rat monoclonal antibody against claudin-4 (1:50). For double immunofluorescence staining, cells were incubated with a mixture of two primary antibodies as follows: rabbit anti-rat polyclonal antibody against $\mathrm{ZO}$ 1 (1:100) were mixed with mouse anti-rat monoclonal antibody against Cav-1 (1:100, Cat.ab17052, Abcam, New Territories, HK), rabbit anti-rat polyclonal antibody against occludin (1:100) were mixed with mouse anti-rat monoclonal antibody against Cav-1 (1:100), and mouse anti-rat monoclonal antibody against claudin-4 (1:50) were mixed with rabbit anti-rat polyclonal 
antibody againstCav-1 (1:100). The primary antibodies were incubated overnight at $4{ }^{\circ} \mathrm{C}$. The cells were then washed and incubated with donkey anti-mouse IgG $(\mathrm{H}$ $+\mathrm{L})$ secondary antibody, Alexa Fluor 594 conjugate (1:100, Invitrogen), and donkey anti-rabbit $\operatorname{IgG}(\mathrm{H}+\mathrm{L})$ secondary antibody, and Alexa Fluor 488 conjugate (1:100, Invitrogen). The cells were washed again and images were obtained using a confocal laser scanning microscope (MTC-600, Bio-Rad, CA, USA) at $800 \times$ magnification.

\section{Transmission electron microscopy}

The TJ structures in the in vitro pulmonary epithelial barrier were examined by transmission electron microscopy (TEM) as described in a previous study [34]. Pulmonary epithelial cells were fixed with $2 \%$ paraformaldehyde and $2 \%$ glutaraldehyde at $4{ }^{\circ} \mathrm{C}$ overnight. After washing with PBS and post-fixation in $1 \%$ osmium tetroxide in phosphate buffer ( $\mathrm{pH}$ 7.4) for $1 \mathrm{~h}$, the cells were dehydrated in a graded ethanol series and embedded in the epoxy resin media. The resin blocks were then cut into ultrathin sections $(60 \mathrm{~nm})$ with a diamond knife, stained with uranyl acetate and lead citrate for contrast, and viewed by TEM (JEM-1200EX, Hitachi Electronic Company, Tokyo, Japan) at $20,000 \times$ or $80,000 \times$ magnification.

\section{Measurement of transepithelial electrical resistance}

Transepithelial electrical resistance (TEER) was measured with a Millicell ERS volt-ohmmeter (Millicell ERS-2,Millipore, MA, USA). The average TEER was calculated by subtracting the average resistance of the cell-free culture inserts and corrected for the area covered by the cell monolayer. Resistance of a unit area $=$ Resistance $(\Omega) \times$ Effective membrane area $\left(\mathrm{cm}^{2}\right)$ [35].

\section{Measurement of cell monolayer permeability}

The permeability of cell monolayers was measured with the apparent permeability coefficient $\left(\mathrm{P}_{\mathrm{app}}\right)$ of fluorescein isothiocyanate-dextran (MW 4400, FD4). Briefly, FD4 (Sigma-Aldrich, St Louis, MA, USA) was directly added to the apical compartments of Transwell inserts (50 $\mathrm{mg} / \mathrm{mL}$ in PBS), and its fluorescence in the basolateral compartments was measured using a spectrofluorometer (F-2000, Hitachi, Tokyo, Japan) every $30 \mathrm{~min}$ for $2 \mathrm{~h}$ at excitation and emission wavelengths of 495 and $515 \mathrm{~nm}$. Hank's buffered salt solution (HBSS, $\mathrm{pH}$ 7.4) was used as the solution in the basolateral compartments. $\mathrm{P}_{\text {app }}$ was calculated as follows:

$$
\text { Papp }=\frac{d Q / d t}{A \times C_{0}}
$$

Where $d Q / d t$ is the FD4 transfer rate, $A$ is the surface area of the Transwell membrane, and $C_{0}$ is the initial concentration of FD4 in the apical compartment [36].

\section{Statistical analysis}

Statistical analysis was performed using SPSS 17.0 software (IBM SPSS Inc., Chicago, USA). Data were presented as the means \pm standard deviation (SD). An unpaired Student's $t$-test was used to determine the significant differences between the different groups. One-way analysis of variance (ANOVA) and Bonferroni tests were used to determine the intragroup significant difference at different time points. A $P$ value of $<0.05$ was considered to be statistically significant.

\section{Results}

Hyperoxia exposure impairs the structure and function of the in vitro pulmonary epithelial barrier

TEM was used to visualize the TJ structures between pulmonary epithelial cells. The normoxic group had normal and intact TJ structures (Fig. 1a and b), while the hyperoxic group exhibited a loss of TJ structures (Fig. 1c) and intermittent widening (Fig. 1d) after $72 \mathrm{~h}$ of exposure.

Both the TEER values after hyperoxia exposure for 48 and $72 \mathrm{~h}$ significantly declined compared with that in the normoxic group $(P<0.05$ for $48 \mathrm{~h}, P<0.01$ for $72 \mathrm{~h}$ ). Prolonged exposure to hyperoxic conditions significantly decreased the TEER value, which reached a lowest value at $72 \mathrm{~h}$ (Fig. 1e).

In line with the decreased TEER values, the Papp of FD4 was higher in the hyperoxic group than in the normoxic group $(P<0.05$ for $48 \mathrm{~h}, P<0.01$ for $72 \mathrm{~h}$ ). Prolonged exposure to hyperoxic conditions significantly increased the Papp of FD4, which peaked at $72 \mathrm{~h}$ (Fig. 1f).

\section{Hyperoxia exposure decreases the expression of $\mathrm{TJ}$ proteins in the pulmonary epithelial barrier}

Compared with the normoxia-treated group, both mRNA and protein levels of ZO-1, occludin, and claudin-4 decreased in the hyperoxic groups after 48 and $72 \mathrm{~h}(P<0.01$ for both levels). In other words, prolonged exposure to hyperoxia significantly decreased both the mRNA (Fig. 2a-d) and protein (Fig. 2e-h) levels of TJ proteins, reaching a lowest value at $72 \mathrm{~h}$ for both levels.

Results of the immunofluorescence staining indicated that in the normoxia-treated group, ZO-1, occludin, and claudin- 4 were localized in the membranes of neighboring epithelial cells showing continuous distribution, while the fluorescence intensity decreased and distribution was discontinuous in the membrane of pulmonary epithelial cells in the $72-\mathrm{h}$ hyperoxic group (Fig. 2i). 

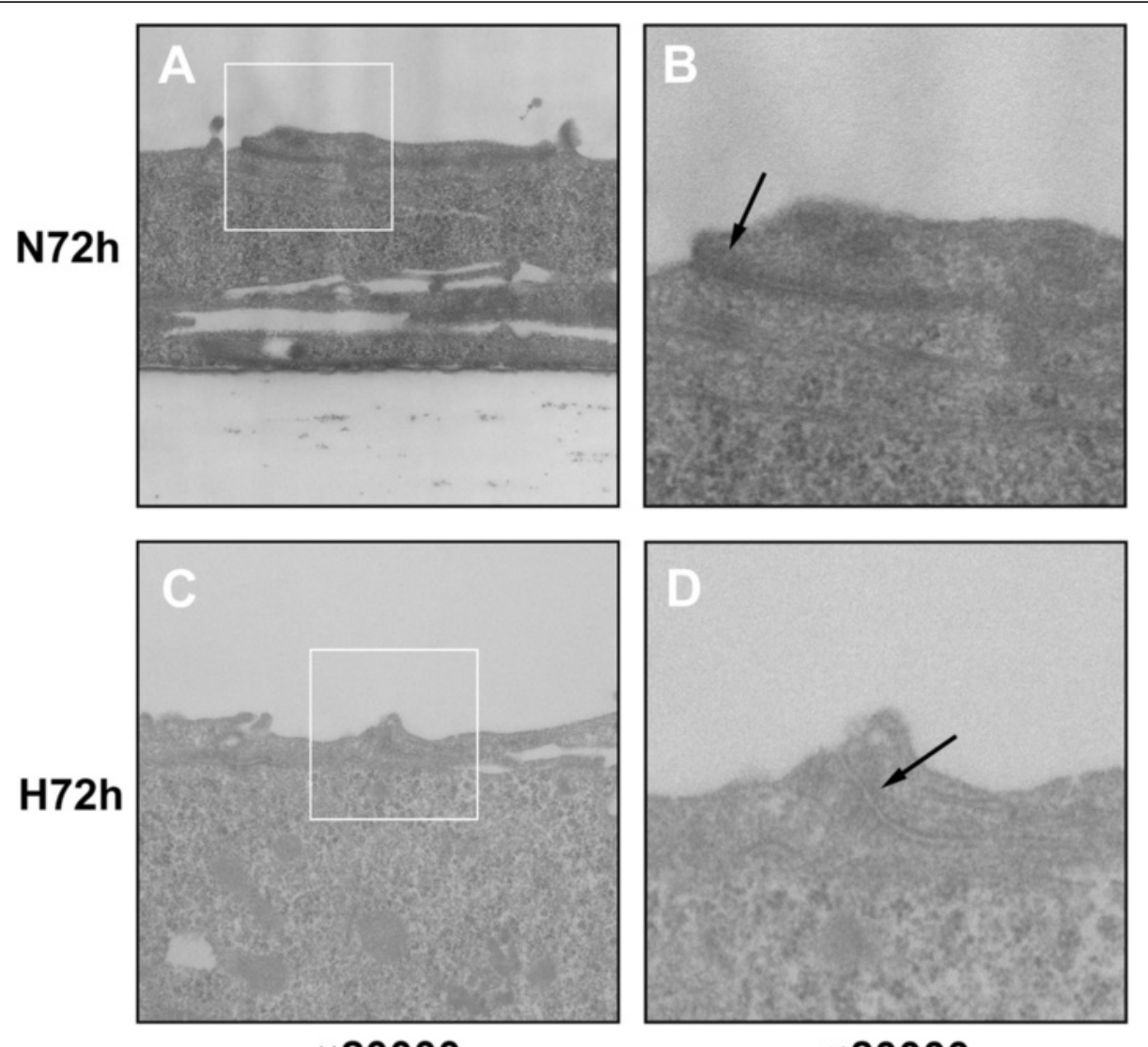

$\times 20000$

$\times 80000$
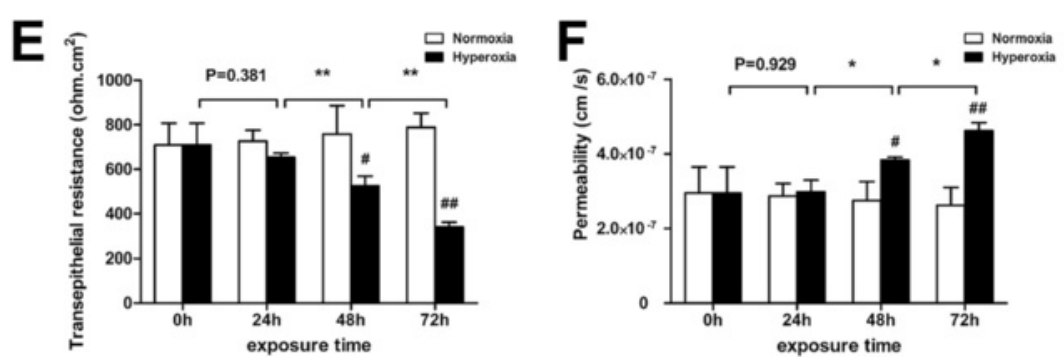

Fig. 1 Effects of hyperoxia exposure on the structure and function of the pulmonary epithelial barrier in vitro. Alveolar cell monolayers were exposed to normoxia (N) (21 \% $\left.\mathrm{O}_{2} / 5 \% \mathrm{CO}_{2}\right)$ or hyperoxia ( $\left.\mathrm{H}\right)\left(85 \% \mathrm{O}_{2} / 5 \% \mathrm{CO}_{2}\right)$ for $0,24,48$, and $72 \mathrm{~h}$. Tight junction structures $(\mathbf{a}, \mathbf{b}, \mathbf{c}, \mathbf{d} ; 20,000 \times$ or $80,000 \times)$ were determined by transmission electron microscopy (TEM). Black arrows indicate the tight junction structures (b, $\mathbf{d})$. Transepithelial electrical resistance (TEER) (e) and apparent permeability coefficient (Papp) of fluorescein isothiocyanate-dextran (FD) (f) were measured. Values are represented as means \pm standard deviation (SD), ${ }^{\#} P<0.05$ and ${ }^{\# \#} P<0.01$ for comparison between the $\mathrm{H}$ and $\mathrm{N}$ groups, ${ }^{*} P<0.05$ and ${ }^{* *} P<0.01$ for comparison between different time points in the $\mathrm{H}$ group

\section{Hyperoxia exposure decreases Cav-1 expression in the pulmonary epithelial barrier}

Compared with the normoxic group, Cav-1 mRNA levels decreased in all three hyperoxia exposure groups $(24,48$, and $72 \mathrm{~h} ; P<0.01$ in all cases); similarly, the Cav-1 protein levels decreased in all three groups $(P<0.05$ for $24 \mathrm{~h}, P<0.01$ for 48 and $72 \mathrm{~h}$ ). Prolonged treatment of hyperoxia significantly decreased both the Cav-1 mRNA (Fig. 3a and b) and protein (Fig. 3c and d) levels, reaching the lowest value at $72 \mathrm{~h}$ for both.

Colocalization of Cav-1 with TJ proteins in the pulmonary epithelial barrier

Double immunofluorescence staining showed that Cav-1 was colocalized with TJ proteins ZO-1 (Fig. 4a and b), occludin (Fig. 4c and d), and claudin-4 (Fig. 4e and $\mathrm{f}$ ) as shown by the yellow fluorescence in the 

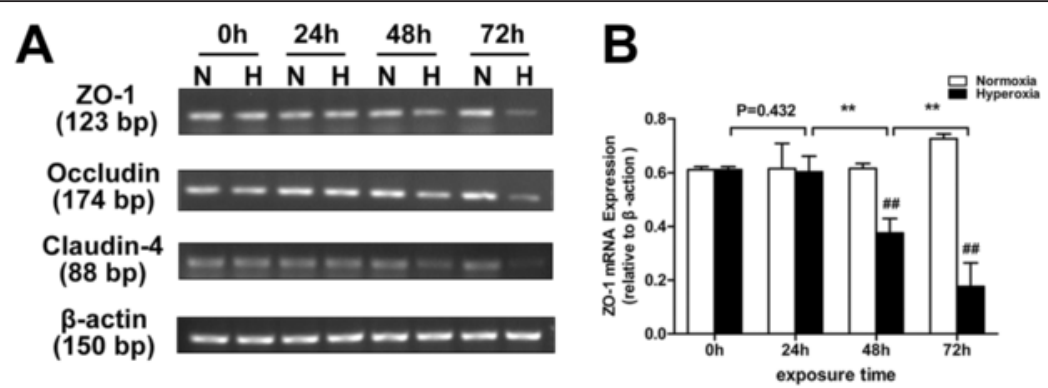

C
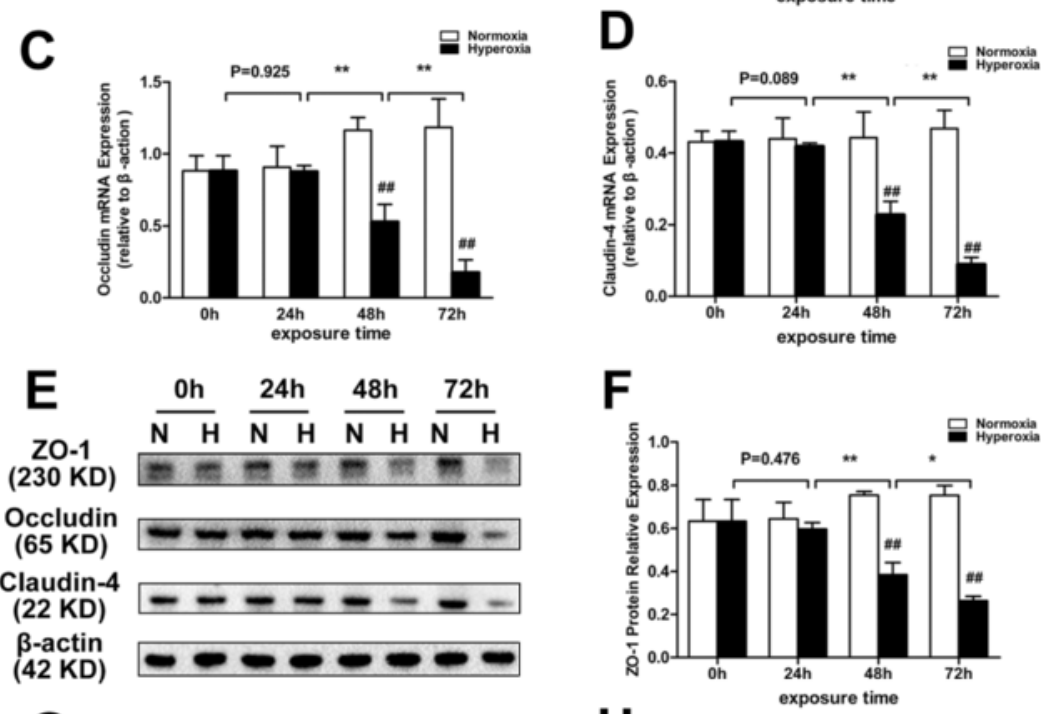

G
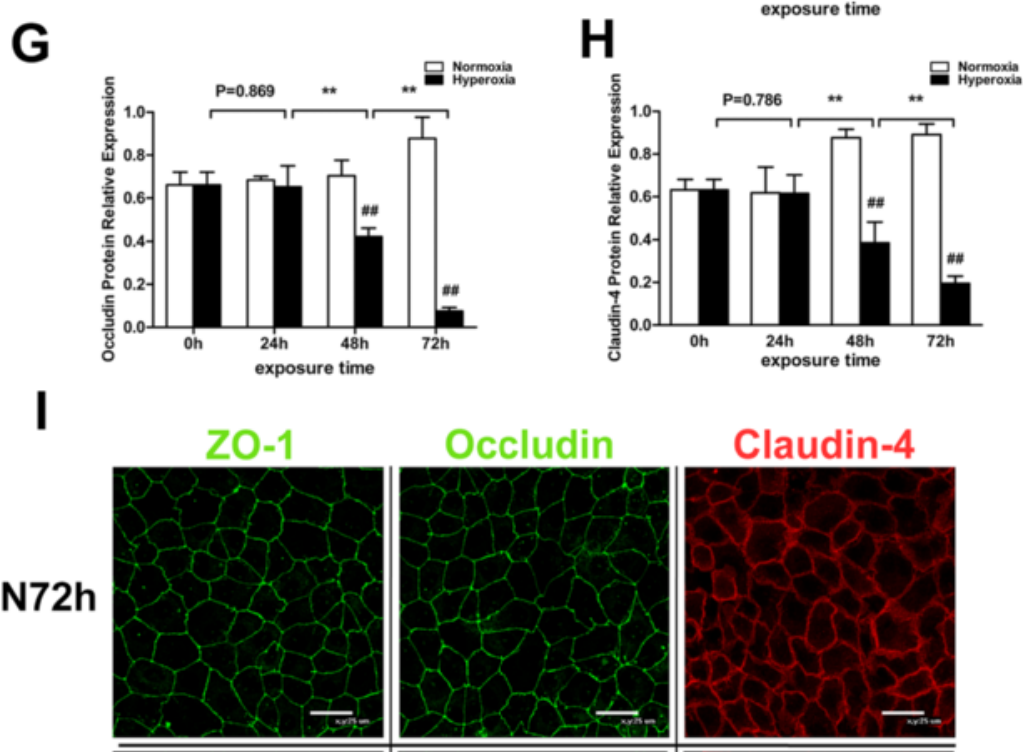

H72h
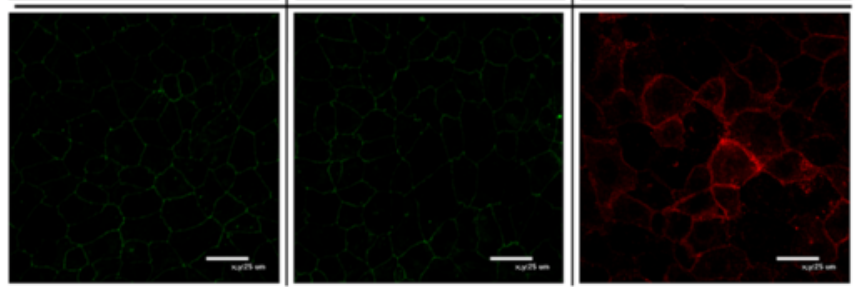

Fig. 2 (See legend on next page.) 
(See figure on previous page.)

Fig. 2 Effects of hyperoxia exposure on the expression and distribution of ZO-1, occludin, and claudin-4 in the in vitro pulmonary epithelial barrier model. Alveolar cell monolayers were exposed to normoxia $(\mathrm{N})\left(21 \% \mathrm{O}_{2} / 5 \% \mathrm{CO}_{2}\right)$ or hyperoxia $(\mathrm{H})\left(85 \% \mathrm{O}_{2} / 5 \% \mathrm{CO}_{2}\right)$ for $0,24,48$, and 72 h. mRNA $(\mathbf{a}, \mathbf{b}, \mathbf{c}, \mathbf{d})$, protein $(\mathbf{e}, \mathbf{f}, \mathbf{g}, \mathbf{h})$, and distribution (i) of ZO-1, occludin, and claudin-4 were determined using RT-PCR, Western blot, and immunofluorescence staining, respectively. (i) Green indicates ZO-1 and occludin, and red indicates claudin-4 (800x magnification). Values are represented as means \pm SD, ${ }^{\# \#} P<0.01$ for comparison between the $\mathrm{H}$ and $\mathrm{N}$ groups, ${ }^{*} \mathrm{P}<0.05$ and ${ }^{* *} \mathrm{P}<0.01$ for comparison between different time points in the $\mathrm{H}$ group

membrane of pulmonary epithelial cells. In the normoxic group, the yellow fluorescence indicating colocalization was strong, while signal intensities of ZO-1, occludin, claudin-4, and Cav-1 were decreased after exposure to hyperoxic conditions for $72 \mathrm{~h}$.

\section{Downregulation of Cav-1 decreases expression of TJ proteins in alveolar epithelial monolayers with normoxia exposure}

Results of the RT-PCR and Western blot analyses showed that Cav-1 mRNA and protein levels were markedly downregulated at $72 \mathrm{~h}$ after transfection with Cav-1-siRNA (Additional file 1: Figure S1A-H). Additional file 3 : Figure S3A-B indicated that the transfection efficiency of Cav-1siRNA was high. Compared with the normoxic and control siRNA groups, the mRNA and protein levels of ZO-1, occludin, and claudin-4 were significantly decreased in the Cav-1-siRNA group $(P<0.01$ for all cases; Fig. $5 a-d)$.

Compared with the normoxic and control siRNA groups, immunofluorescence staining showed a decreased fluorescence level and discontinuous distribution of ZO-1/occludin (green) and claudin-4 (red) in the Cav-1-siRNA group (Fig. 5e).
Downregulation of Cav-1 disrupts the structure and function of alveolar epithelial monolayers with normoxia exposure

Compared with the epithelial barrier cells of the normoxic group, which showed an intact $\mathrm{TJ}$ structure (Fig. 6a and b), the TJ structure was disrupted and intermittently widened between neighboring epithelial cells after Cav-1 knockdown (Fig. 6c and d).

In line with the structural disruption, Cav-1-siRNA transfection resulted in a significant reduction in the TEER value by 40 and $37 \%$ and significant increase in the Papp of FD 4 by 48 and $42 \%$, compared with the normoxic and the control siRNA groups, respectively (all $P<0.01$; Fig. 6e and $\mathrm{f}$ ).

\section{Upregulating Cav-1 restores expression of TJ proteins in alveolar epithelial monolayers exposed to hyperoxia} Results of the RT-PCR and Western blot analysis showed that Cav-1 mRNA and protein levels were markedly upregulated at $48 \mathrm{~h}$ after transfection with Cav-1 cDNA (Additional file 2: Figure S2A-H). Additional file 3: Figure S3C-F indicated that the transfection efficiency of pCMV6-CAV1 under normoxic or hyperoxic condition

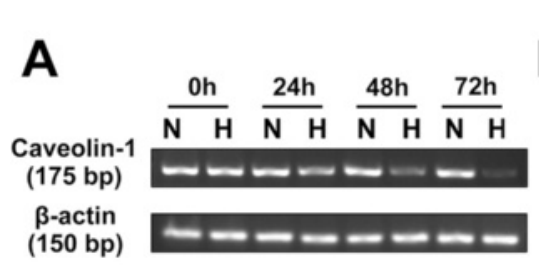

B
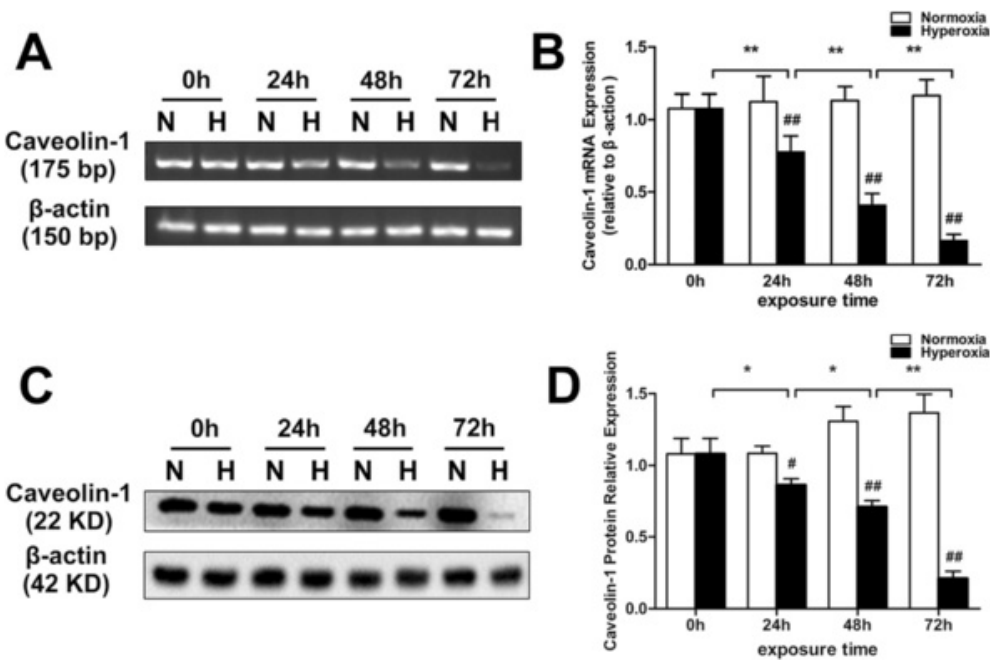

Fig. 3 Effects of hyperoxia exposure on the mRNA and protein expression of Cav-1 in an in vitro pulmonary epithelial barrier model. Alveolar cell monolayers were exposed to normoxia ( $\mathrm{N})\left(21 \% \mathrm{O}_{2} / 5 \% \mathrm{CO}_{2}\right)$ or hyperoxia $(\mathrm{H})\left(85 \% \mathrm{O}_{2} / 5 \% \mathrm{CO}_{2}\right)$ for 0, 24, 48, and $72 \mathrm{~h}$. Cav-1 mRNA (a, b) and protein $(\mathbf{c}, \mathbf{d})$ levels were monitored with RT-PCR and Western blot analysis, respectively. Values are represented as means \pm SD, ${ }^{\#} P<0.05$ and ${ }^{\# \#} P$ $<0.01$ for comparison between the $\mathrm{H}$ and $\mathrm{N}$ groups, ${ }^{*} \mathrm{P}<0.05$ and ${ }^{* *} \mathrm{P}<0.01$ for comparison between different time points in the $\mathrm{H}$ group 


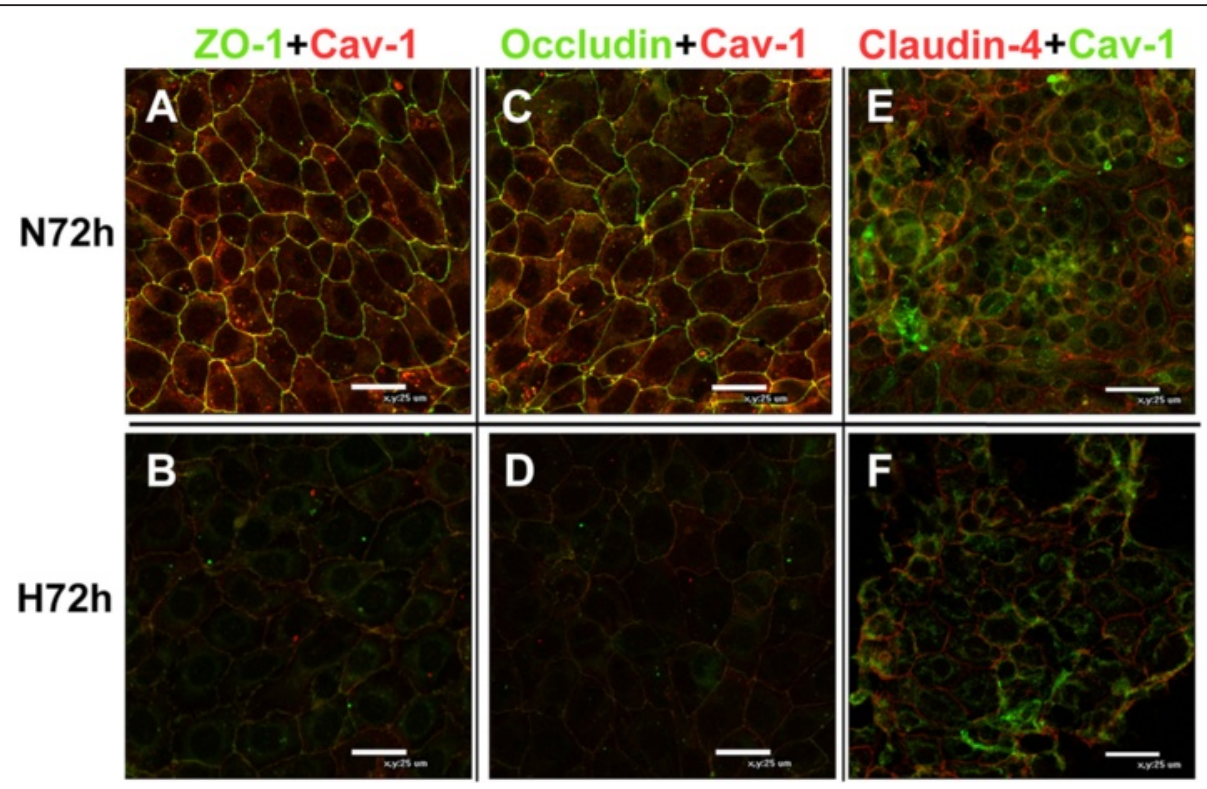

Fig. 4 Colocalization of Cav-1 and tight junction proteins in an in vitro pulmonary epithelial barrier model. Alveolar cell monolayers were exposed to normoxia ( $\mathrm{N})\left(21 \% \mathrm{O}_{2} / 5 \% \mathrm{CO}_{2}\right)$ or hyperoxia $(\mathrm{H})\left(85 \% \mathrm{O}_{2} / 5 \% \mathrm{CO}_{2}\right)$ for $72 \mathrm{~h}$. The colocalization of Cav-1 and tight junction proteins in the pulmonary epithelium in vitro was monitored by double immunofluorescence staining. a-d green represents ZO-1 and occludin, respectively, and red represents Cav-1. e and $\mathbf{f}$, green represents Cav-1 and red represents claudin-4. Yellow represents the colocalization of Cav-1 and tight junction proteins (800× magnification)

was high. Compared with the hyperoxic and empty vectortransfected groups, upregulating Cav-1 expression increased both the mRNA and protein levels of ZO-1, occludin, and claudin-4 $(P<0.01$ in all cases; Fig. $7 \mathrm{a}-\mathrm{d})$ under hyperoxic conditions. Accordingly, immunofluorescence staining results also showed that the distribution and fluorescence intensities of ZO-1, occludin, and claudin-4 under hyperoxic conditions were rescued (Fig. 7e) after Cav-1 cDNA transfection.

\section{Upregulation of Cav-1 rescues structural and functional destruction of pulmonary epithelial barrier under hyperoxic conditions}

The disruption in the TJ structure of the pulmonary epithelial barrier (Fig. 8a and b) caused by hyperoxic conditions was partially rescued and the intermittent widening was narrowed (Fig. 8c and d) after Cav-1 cDNA transfection.

In line with the structural rescue, after Cav-1 cDNA transfection, the TEER value significantly increased by 34 and $39 \%$ and the Papp of FD4 significantly decreased by 29 and $26 \%$, compared with the hyperoxic and empty vector groups, respectively $(P<0.05$ in all cases; Fig. $8 \mathrm{e}$ and $\mathrm{f})$.

\section{Discussion}

BPD is a common complication in preterm infants with birth weight less than 1500 g. Currently, mechanisms underlying pulmonary edema at an early stage of BPD mainly involve increased permeability of lung vasculature induced by inflammatory factors [6,37], defects in pulmonary fluid clearance induced by aquaporin-1 and amiloride-sensitive sodium channel upregulation on the surface of AEC-II [38], and disruption of the pulmonary vascular endothelial barrier induced by downregulation of connexin proteins [8]. In our previous study, we used a hyperoxia-induced BPD model and found destruction of pulmonary epithelial TJ structures, increased permeability of the barrier, and increased wet/dry ratio of lung tissues, indicating that the destruction of the pulmonary epithelial TJ structures is a key step during the pathogenesis of pulmonary edema in BPD [7]. The results of the in vitro pulmonary epithelial barrier model exposed to hyperoxic conditions in this study are consistent with the in vivo results of our previous study. Transepithelial resistance was found to be decreased under hyperoxic exposure, which is also consistent with some in vitro experiments in which hyperoxia was found to decrease the transepithelial resistance in 16HBE14 cell and Calu-3 cell monolayers [39].

$\mathrm{TJ}$ is a protein complex located at the apical side of cell-cell junctions. It seals the space between neighboring cells and forms an important barrier to maintain cell polarity and modulate paracellular fluid transportation [10]. Ions, small molecules, and immune cells can transfer through the barrier and induce edema when $\mathrm{T}$ ) structures are broken $[40,41]$. Downregulation or mislocalization of TJ proteins is known to result in pulmonary epithelial barrier destruction [13, 14]. Here, ZO-1, 


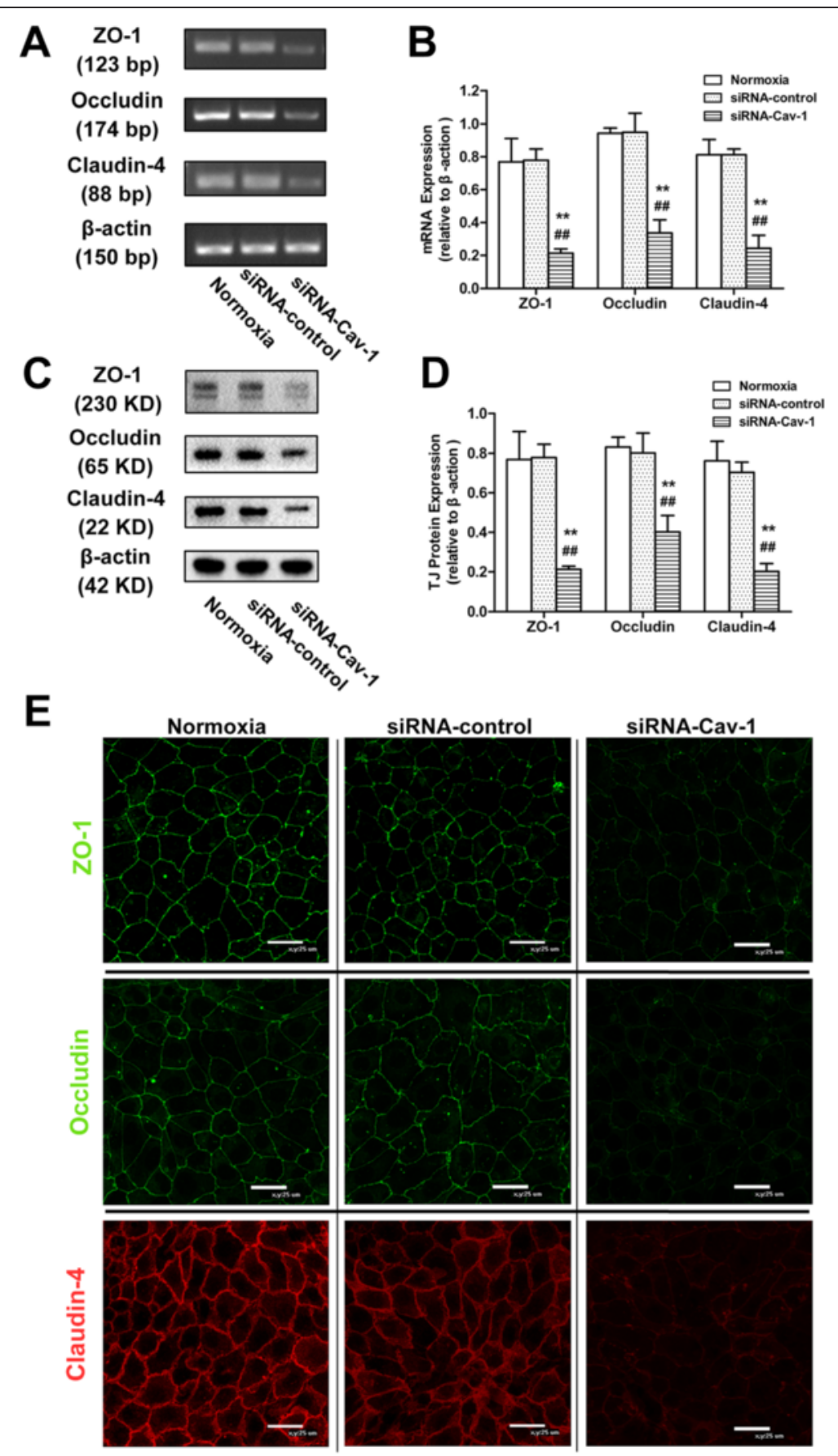

Fig. 5 Effects of Cav-1 downregulation on the expression and distribution of ZO-1, occludin, and claudin-4 in alveolar epithelial monolayers with normoxia exposure. At 60-70 \% confluence, alveolar epithelial cells were treated with normoxia exposure, control siRNA transfection, and siRNA-Cav-1 transfection $(10 \mathrm{~nm})$ for 72 h. mRNA (a, b), protein $(\mathbf{c}, \mathbf{d})$, and distribution (e) of ZO-1, occludin, and claudin-4 were monitored by RT-PCR, Western blot, and immunofluorescence staining, respectively. (e) Green represents ZO-1 and occludin, and red represents claudin-4 (800x magnification). Values are represented as the means $\pm S D,{ }^{\#} P<0.01$ for comparison with the normoxia-treated group and ${ }^{* *} P<0.01$ for comparison with the control siRNA-transfected group

occludin, and claudin-4 mRNA and protein levels were found to decrease under hyperoxic conditions. Moreover, the maximum decreases in the levels of these molecules matched the maximum destruction of the pulmonary epithelial barrier structure and function, indicating that the downregulation of $\mathrm{ZO}-1$, occludin, and claudin-4 is responsible for the hyperoxia-induced destruction of the pulmonary epithelial barrier. These are 

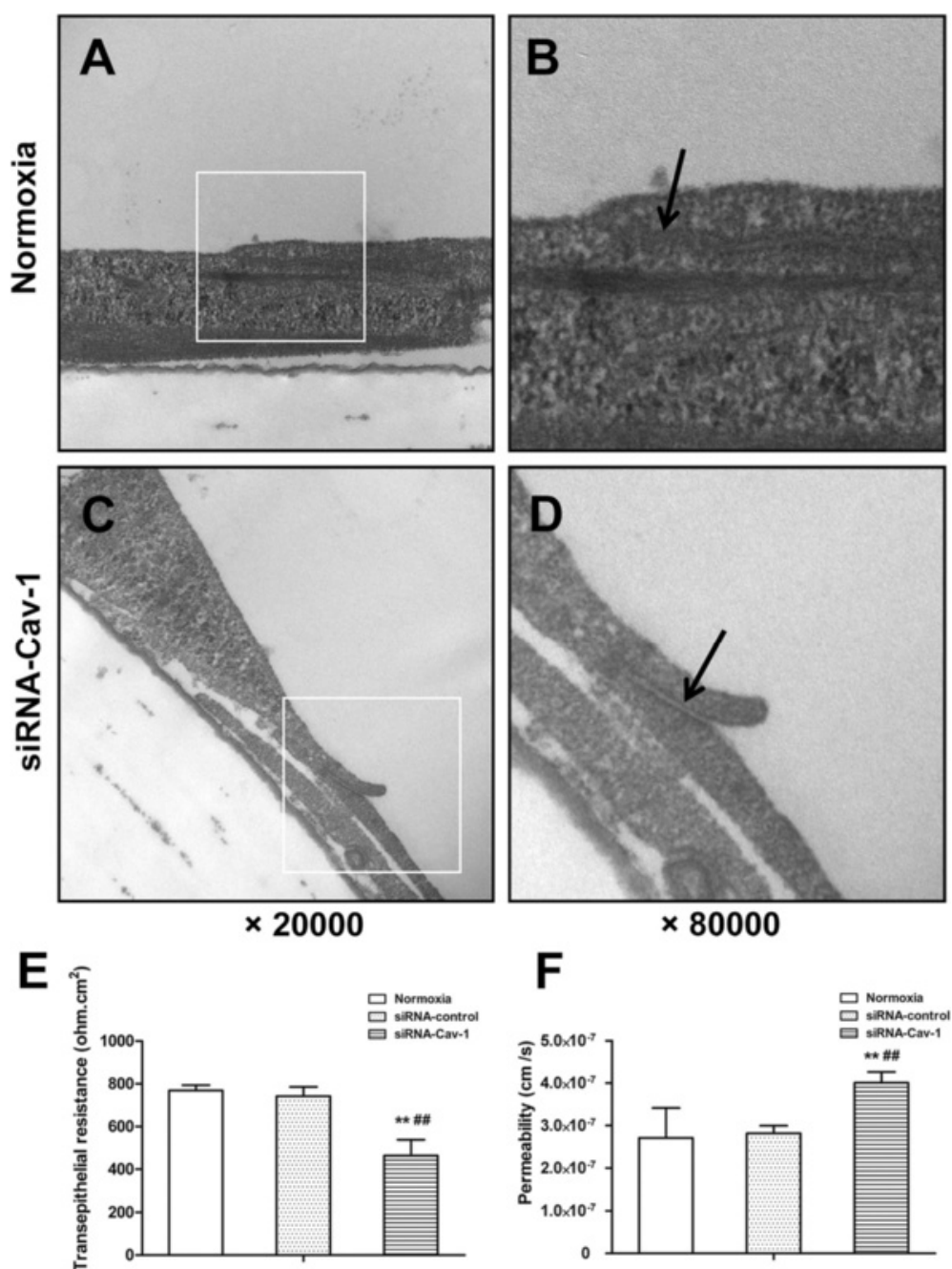

Fig. 6 Effects of Cav-1 downregulation on the structure and function of alveolar epithelial monolayers exposed to normoxic conditions. At 60$70 \%$ confluence, alveolar epithelial cells were exposed to normoxic conditions, control siRNA transfection, and siRNA-Cav-1 transfection (10 nm) for $72 \mathrm{~h}$. Tight junction structures ( $\mathbf{a}, \mathbf{b}, \mathbf{c}, \mathbf{d}, 20,000 \times$ or $80,000 \times$ magnification) were determined by TEM. Black arrows indicate the tight junction structures $(\mathbf{b}, \mathbf{d})$. The TEER value $(\mathbf{e})$ and Papp of FD4 (f) were also measured. Values are represented as means \pm SD, ${ }^{\# \#} P<0.01$ for comparison with the normoxia-treated group, ${ }^{* *} P<0.01$ for comparison with the control siRNA-transfected group

consistent with the in vivo results of our previous study [7].

Cav-1 is a scaffold protein with a scaffolding domain that can interact with multiple downstream signaling molecules and modulate their activities [24, 25]. Cav-1 is downregulated in several lung diseases such as asthma, chronic obstructive pulmonary disorder, and idiopathic pulmonary fibrosis [42]. In a fetal sheep lung injury model induced by in utero LPS injection, it was found that the Cav-1 gene and protein levels both decreased, and the Smad2/3, Stat, and a-SMase/ceramide signaling pathways were activated, indicating that the downregulation of Cav-1 and activation of these signaling pathways may together contribute to the occurrence of BPD [30]. In our present study, both in the hyperoxia-induced BPD model in the newborn rats (data not shown) and the in vitro pulmonary epithelial barrier model exposed to hyperoxic conditions, Cav-1 mRNA and protein levels were decreased, which is consistent with the results of the previous study [30]. Moreover, exposure to hyperoxic conditions resulted in the downregulation of Cav-1 gene transcription and protein expression levels (at $24 \mathrm{~h}$ after hyperoxia exposure), which preceded the downregulation of ZO-1, occludin, and claudin-4 expression at both the mRNA and protein levels (at $48 \mathrm{~h}$ after hyperoxia exposure). In addition, Cav-1 colocalizes with the other $3 \mathrm{TJ}$ proteins in the membrane of 

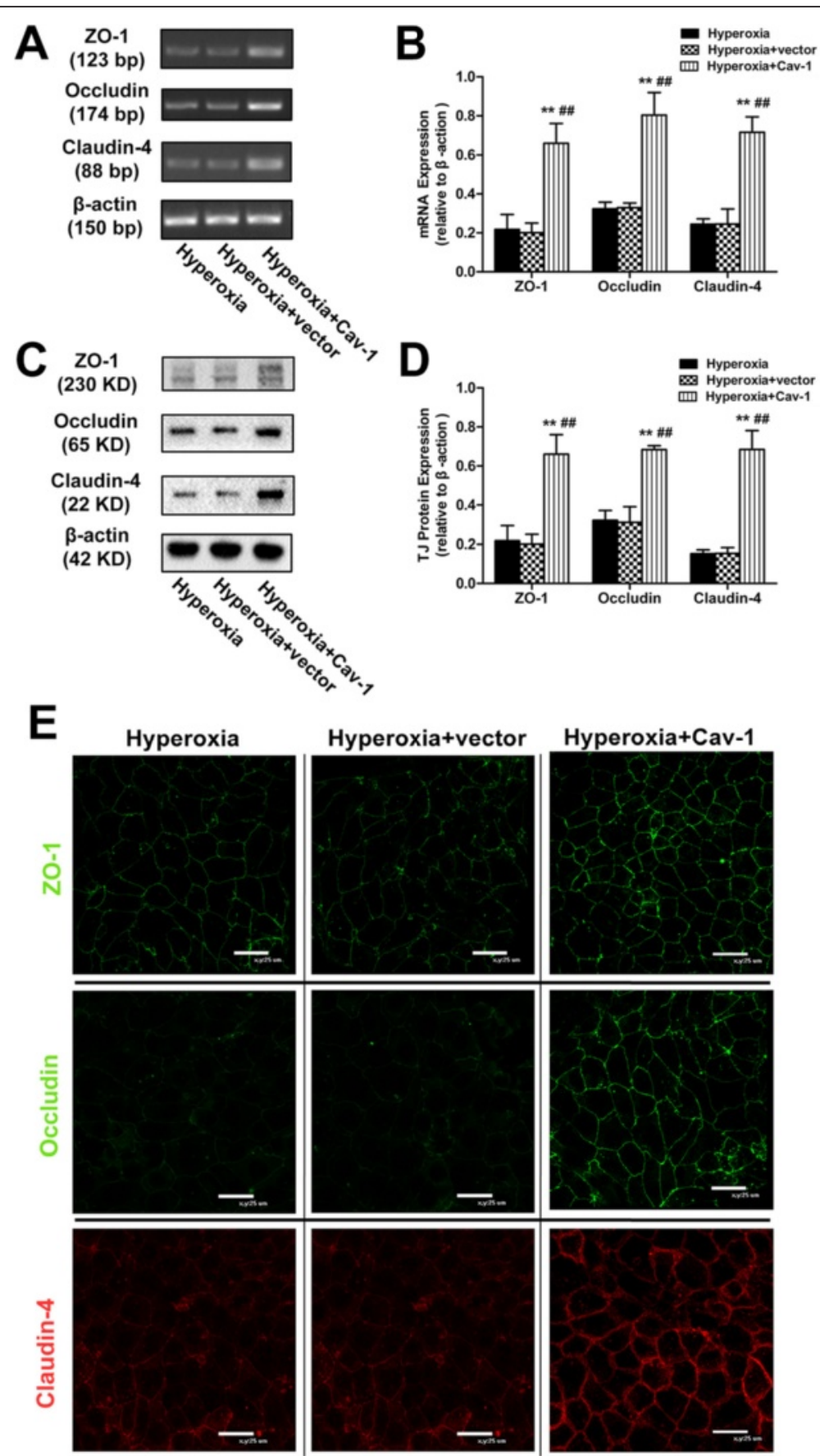

Fig. 7 Effects of Cav-1 upregulation on the expression and distribution of ZO-1, occludin, and claudin-4 in alveolar epithelial monolayers exposed to hyperoxic conditions. At 60-70 \% confluence, alveolar epithelial cells were treated with empty vector and Cav-1 cDNA (1 $\mu \mathrm{gg} / \mathrm{mL}) \mathrm{transfection}$ and then immediately exposed to hyperoxic conditions for $48 \mathrm{~h}$. mRNA $(\mathbf{a}, \mathbf{b})$, protein (c, $\mathbf{d})$, and distribution (e) of ZO-1, occludin, and claudin-4 were monitored by RT-PCR, Western blot, and immunofluorescence staining respectively. (e) Green represents ZO-1 and occludin, and red represents claudin-4 (800x magnification). Values are represented as means $\pm \mathrm{SD},{ }^{\# \#} P<0.01$ for comparison with the hyperoxia-treated group, ${ }^{* * *} P<0.01$ for comparison with the empty vector-transfected group

pulmonary epithelial cells, which is consistent with some previous studies showing colocalization between Cav-1 and occludin [37]. These results reveal the existence of temporal and spatial correlations between
Cav-1 and TJ proteins in the in vitro pulmonary epithelial barrier model with hyperoxia exposure.

In 2000, Nusrat and colleagues found that Cav-1 may modulate the assembly of $\mathrm{TJ}$ proteins [26]. In some 

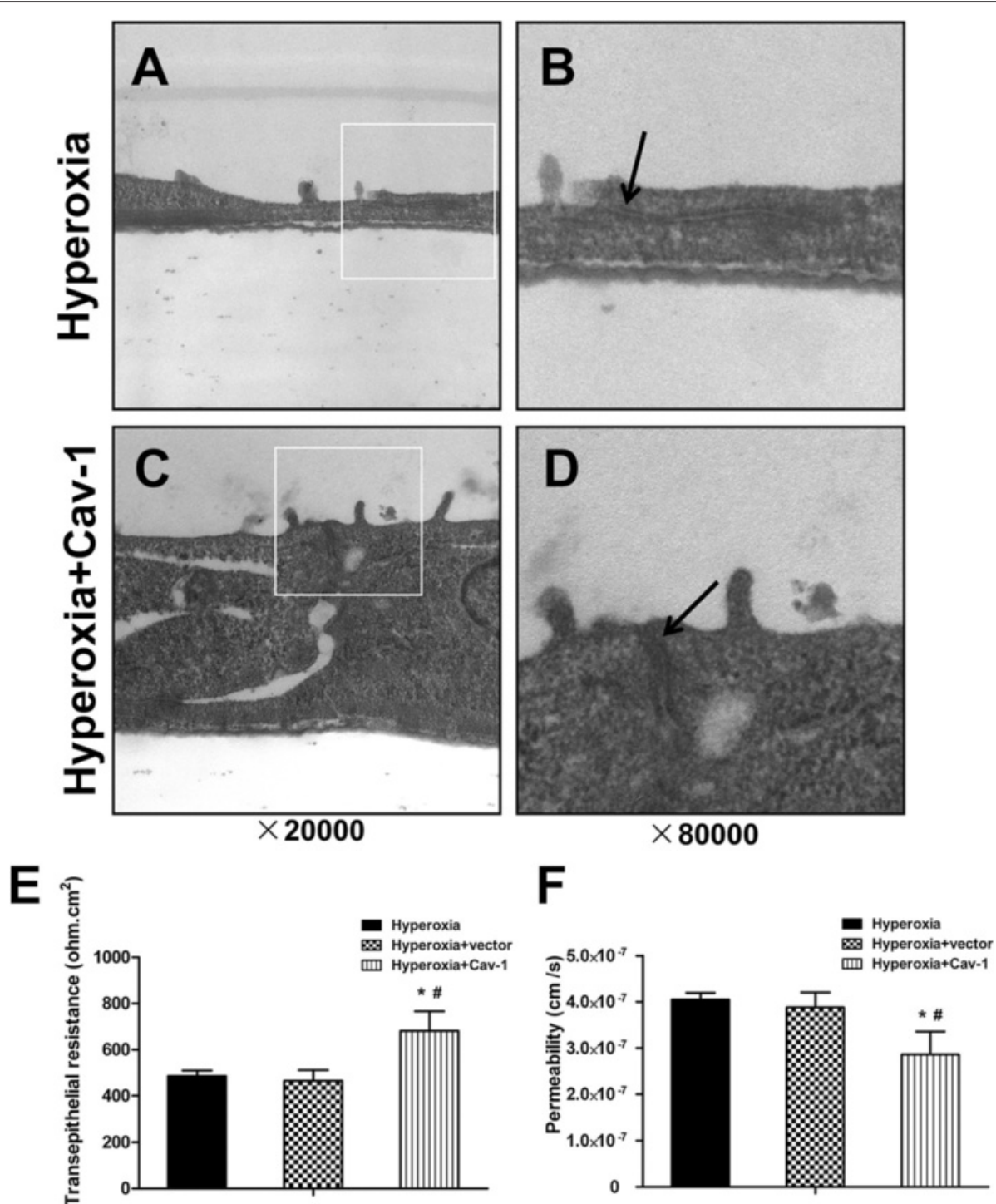

Fig. 8 Effects of Cav-1 upregulation on the structure and function of rat alveolar epithelial monolayers exposed to hyperoxic conditions. At 60$70 \%$ confluence, alveolar epithelial cells were treated with empty vector and Cav-1 CDNA (1 $\mathrm{\mu g} / \mathrm{mL})$ transfection and then immediately exposed to hyperoxic conditions for $48 \mathrm{~h}$. Tight junction structures ( $\mathbf{a}, \mathbf{b}, \mathbf{c}, \mathbf{d}, 20,000 \times$ or $80,000 \times$ magnification) were determined by TEM. Black arrows indicate the tight junction structures $(\mathbf{b}, \mathbf{d})$. TEER value $(\mathbf{e})$ and Papp of FD4 (f) were also measured. Values are presented as means \pm SD, ${ }^{\#} P<0.05$ for comparison with the hyperoxia-treated group, ${ }^{*} P<0.05$ for comparison with the empty vector-transfected group

disease models and cell lines, Cav-1 and TJ protein expression levels were either positively or negatively correlated. For instance, in a rat cortical cold injury model, the increase in Cav-1 was found to precede the decrease in the claudin-5 and occludin levels [43]. Contrarily, Song et al. found that downregulation of Cav-1 in brain microvascular endothelial cells using siRNA resulted in a concomitant decrease in the expression levels of ZO-1 and occludin [27]. Brott et al. found that ZO-1 and claudin-4 were downregulated in a rat mesenteric artery injury model induced by fenoldopam, which may have caused by the Cav-1 downregulation by the activation of nitric oxide (NO) signaling pathway [44]. In the present study, we found that Cav-1 downregulation in the pulmonary epithelial barrier model not only decreased the ZO-1, occludin, and claudin-4 mRNA and protein levels, but also disrupted the structure and function of the pulmonary epithelial barrier. Together with previous findings, our findings indicate that Cav-1 downregulation-mediated loss of TJ proteins may be the main cause of pulmonary epithelial barrier destruction induced by hyperoxia.

How Cav-1 positively regulates the expression of $\mathrm{TJ}$ proteins remains to be fully understood. It may be related to the activation of Src tyrosine kinases and MMPs. 
Src tyrosine kinases can be negatively regulated by the scaffolding domain of Cav-1 [45]. A recent study showed that the Src kinase inhibitor SU6656 can rescue the destruction of bronchial epithelial barrier and the expression of occludin [46]. Therefore, it is quite possible that Src tyrosine kinases participate in the process by which Cav-1 regulates the expression of TJ proteins. Second, some previous work showed that Cav-1 can negatively regulate MMPs [47, 48]. The activation of MMPs can lead to the degradation of $\mathrm{TJ}$ proteins in epithelial barriers $[49,50]$. Vermeer et al. found that MMP-9 activation decreased occludin and claudin-1 in respiratory epithelium in human [20]. The downregulation of Cav-1 may lead to the activation of MMPs and further degradation of TJ proteins. However, these assumptions need further verification.

Moreover, cavtratin, a synthetic peptide with the same sequence as the scaffolding domain of Cav-1, can rescue the heightened permeability of the barrier. Cavtratin can inhibit the vascular leakage induced by carrageenan [51], decrease permeability of tumor microvasculature [52], attenuate heightened permeability of blood-brain barrier induced by monocyte chemoattractant protein-1 [27], and attenuate heightened permeability of mesenteric vessel induced by platelet-activating factor [53]. In the present study, Cav-1 upregulation was found to not only rescue the expression of $\mathrm{TJ}$ proteins but also to attenuate the structure and function of the in vitro pulmonary epithelial barrier exposed to hyperoxic conditions.

\section{Conclusions}

In summary, to our knowledge, this is the first study to establish that the Cav-1-mediated downregulation of TJ proteins could be a key cause for the destruction of the pulmonary epithelial barrier when exposed to hyperoxic conditions. These results shed light into the mechanism underlying pulmonary epithelial barrier destruction during hyperoxia-induced BPD. The study suggests that Cav-1 may be a novel potential target for preventing pulmonary edema at an early stage of BPD.

\section{Additional files}

Additional file 1: Figure S1. Effects of transfection concentration and transfection time of Cav-1 siRNA on mRNA and protein expression of Cav-1 in alveolar cell monolayers. At $72 \mathrm{~h}$ after transfection of Cav-1siRNA at a final concentration of $10 \mathrm{~nm}$, significant reductions in the caveolin-1 mRNA ( $A, B, E, F)$, and protein $(C, D, G, H)$ levels were readily apparent. mRNA and protein expressions were determined by RT-PCR and Western blot analysis, respectively. $\beta$-actin was used as an internal control. Values are represented as means $\pm S D, \# \#<0.01$ for comparison between the normoxia and siRNA-CAV1-transfected group, ${ }^{* *} P<0.01$ for comparison between different concentration transfected groups or different time ransfected groups. (TIF $1844 \mathrm{~kb}$ )

Additional file 2: Figure S2. Effects of transfection concentration and transfection time of Cav-1 cDNA on mRNA and protein expression of
Cav-1 in alveolar cell monolayers. At $48 \mathrm{~h}$ after transfection of $1 \mathrm{\mu g} / \mathrm{mL}$ Cav-1 cDNA, significant increases in caveolin-1 mRNA (A, B, E, F) and protein $(C, D, G, H)$ were readily apparent. mRNA and protein expressions were determined by RT-PCR and Western blot analysis, respectively. $\beta$-actin was used as an internal control. Values are represented as means $\pm S D,{ }^{\# \#} P<$ 0.01 for comparison between the normoxia and CAV1 CDNA-transfected group, ${ }^{* * *} P<0.01$ for comparison between different concentration transfected groups or different time ransfected groups. (TIF $1801 \mathrm{~kb}$ )

Additional file 3: Figure S3. Efficacy assessment of Cav-1-siRNA transfection and PCMV6-CAV1 transfection. At 60-70 \% confluence, cells exposed to normoxia were transfected with $10 \mathrm{~nm}$ of a control siRNA (Fluorescein Conjugate) (Cat.\#6201;Cell Signaling Danvers MA) for $72 \mathrm{~h}$ to assess transfection efficiency for Cav-1-siRNA, while cells exposed to normoxia or hyperoxia were transfected with $1 \mu \mathrm{g} / \mathrm{mL}$ of PCMV6-AC-GFP (Cat.\#PS100010; OriGene Technologies) for $48 \mathrm{~h}$ to assess transfection efficiency for pCMV6-CAV1. Images were obtained using a confocal laser scanning microscope at 100x magnification. Green represents transfected cells. The areas of transfected cells and all the cells in the view were measured by image pro plus software, and the ratio represents transfection efficiency. The transfection efficiency of control siRNA was $(88.29 \pm 8.25) \%$

$(A, B)$. The transfection efficiency of pCMV6-AC-GFP under normoxic conditions was $(92.81 \pm 4.16) \%(C, D)$. The transfection efficiency of pCMV6AC-GFP under hyperoxic conditions was $(90.42 \pm 6.83) \%(E$, F). (TIF $10078 \mathrm{~kb})$

\section{Abbreviations}

AEC-II: type II alveolar epithelial cells; BPD: bronchopulmonary dysplasia; BSA: bovine serum albumin; Cav-1: caveolin-1; FBS: fetal bovine serum; PVDF: polyvinylidene difluoride; RT-PCR: reverse transcription-polymerase chain reaction; SD: standard deviation; SDS: sodium dodecyl sulfate; SPC: surfactant protein C; TEER: transepithelial electrical resistance;

TEM: transmission electron microscopy; TJ: tight junction.

\section{Competing interests}

The authors declare that they have no competing interests.

\section{Author contribution}

SY Xu did all experiments and wrote the paper. JH Fu and XD Xue generated the original idea, designed the experiments, and modified the paper. K You assisted in the RT-PCR analyses. All authors read and approved the final manuscript.

\section{Acknowledgements}

This work was funded by the Natural Science Foundation of China (No: 81170605 and No: 81471489).

Received: 7 August 2015 Accepted: 25 April 2016

Published online: 12 May 2016

\section{References}

1. Jobe AH, Bancalari E. Bronchopulmonary dysplasia. Am J Respir Crit Care Med. 2001;163:1723-9.

2. Chess PR, D'Angio CT, Pryhuber GS, Maniscalco WM. Pathogenesis of bronchopulmonary dysplasia. Semin Perinatol. 2006;30:171-8.

3. Doyle LW, Faber B, Callanan C, Freezer N, Ford GW, Davis NM. Bronchopulmonary dysplasia in very low birth weight subjects and lung function in late adolescence. Pediatrics. 2006;118:108-13.

4. Bhandari A, McGrath-Morrow S. Long-term pulmonary outcomes of patients with bronchopulmonary dysplasia. Semin Perinatol. 2013;37:132-7.

5. Anderson PJ, Doyle LW. Neurodevelopmental outcome of bronchopulmonary dysplasia. Semin Perinatol. 2006;30:227-32.

6. Ji W, Fu J, Nie H, Xue X. Expression and activity of epithelial sodium channel in hyperoxia-induced bronchopulmonary dysplasia in neonatal rats. Pediatr Int. 2012;54:735-42.

7. You K, Xu X, Fu J, Xu S, Yue X, Yu Z, et al. Hyperoxia disrupts pulmonary epithelial barrier in newborn rats via the deterioration of occludin and ZO-1. Respir Res. 2012;13:36.

8. Li C, Fu J, Liu H, Yang H, Yao L, You K, et al. Hyperoxia arrests pulmonary development in newborn rats via disruption of endothelial tight junctions and downregulation of Cx40. Mol Med Rep. 2014;10:61-7. 
9. Husain AN, Siddiqui NH, Stocker JT. Pathology of arrested acinar development in postsurfactant bronchopulmonary dysplasia. Hum Pathol. 1998:29:710-7.

10. Gonzalez-Mariscal L, Betanzos A, Nava P, Jaramillo BE. Tight junction proteins. Prog Biophys Mol Biol. 2003;81:1-44.

11. Balda MS, Matter K. The tight junction protein ZO-1 and an interacting transcription factor regulate ErbB-2 expression. EMBO J. 2000;19:2024-33.

12. Neuhaus W, Samwer F, Kunzmann S, Muellenbach RM, Wirth M, Speer CP, et al. Lung endothelial cells strengthen, but brain endothelial cells weaken barrier properties of a human alveolar epithelium cell culture model. Differentiation. 2012;84:294-304.

13. Caraballo JC, Yshii C, Westphal W, Moninger T, Comellas AP. Ambient particulate matter affects occludin distribution and increases alveolar transepithelial electrical conductance. Respirology. 2011;16:340-9.

14. Caraballo JC, Yshii C, Butti ML, Westphal W, Borcherding JA, Allamargot C, et al. Hypoxia increases transepithelial electrical conductance and reduces occludin at the plasma membrane in alveolar epithelial cells via PKC-zeta and PP2A pathway. Am J Physiol Lung Cell Mol Physiol. 2011;300:L569-78.

15. Bazzoni G, Martinez-Estrada OM, Orsenigo F, Cordenonsi M, Citi S, Dejana E. Interaction of junctional adhesion molecule with the tight junction components ZO-1, cingulin, and occludin. J Biol Chem. 2000;275:20520-6.

16. Clark CA, Thomas LK, Azghani AO. Inhibition of protein kinase $C$ attenuates Pseudomonas aeruginosa elastase-induced epithelial barrier disruption. Am J Respir Cell Mol Biol. 2011;45:1263-71.

17. Mitchell LA, Overgaard CE, Ward C, Margulies SS, Koval M. Differential effects of claudin-3 and claudin-4 on alveolar epithelial barrier function. Am J Physiol Lung Cell Mol Physiol. 2011;301:L40-9.

18. Wray C, Mao Y, Pan J, Chandrasena A, Piasta F, Frank JA. Claudin-4 augments alveolar epithelial barrier function and is induced in acute lung injury. Am J Physiol Lung Cell Mol Physiol. 2009;297:L219-27.

19. Coyne CB, Vanhook MK, Gambling TM, Carson JL, Boucher RC, Johnson LG. Regulation of airway tight junctions by proinflammatory cytokines. Mol Biol Cell. 2002;13:3218-34.

20. Vermeer PD, Denker J, Estin M, Moninger TO, Keshavjee S, Karp P, et al. MMP9 modulates tight junction integrity and cell viability in human airway epithelia. Am J Physiol Lung Cell Mol Physiol. 2009;296:L751-62.

21. Cichon C, Sabharwal H, Ruter C, Schmidt MA. MicroRNAs regulate tight junction proteins and modulate epithelial/endothelial barrier functions. Tissue Barriers. 2014;2:e944446.

22. Citalan-Madrid AF, Garcia-Ponce A, Vargas-Robles H, Betanzos A, Schnoor M. Small GTPases of the Ras superfamily regulate intestinal epithelial homeostasis and barrier function via common and unique mechanisms. Tissue Barriers. 2013;1:e26938.

23. Suzuki T, Elias BC, Seth A, Shen L, Turner JR, Giorgianni F, et al. PKC eta regulates occludin phosphorylation and epithelial tight junction integrity. Proc Natl Acad Sci U S A. 2009;106:61-6.

24. Hommelgaard AM, Roepstorff K, Vilhardt F, Torgersen ML, Sandvig K, van Deurs B. Caveolae: stable membrane domains with a potential for internalization. Traffic. 2005:6:720-4.

25. Insel PA, Head BP, Ostrom RS, Patel HH, Swaney JS, Tang CM, et al. Caveolae and lipid rafts: $G$ protein-coupled receptor signaling microdomains in cardiac myocytes. Ann N Y Acad Sci. 2005;1047:166-72.

26. Nusrat A, Parkos CA, Verkade P, Foley CS, Liang TW, Innis-Whitehouse W, et al. Tight junctions are membrane microdomains. J Cell Sci. 2000;113(Pt 10): 1771-81.

27. Song L, Ge S, Pachter JS. Caveolin-1 regulates expression of junction-associated proteins in brain microvascular endothelial cells. Blood. 2007:109:1515-23.

28. Marchiando AM, Shen L, Graham WV, Weber CR, Schwarz BT, Austin 2nd JR, et al. Caveolin-1-dependent occludin endocytosis is required for TNFinduced tight junction regulation in vivo. J Cell Biol. 2010;189:111-26.

29. Jin Y, Lee SJ, Minshall RD, Choi AM. Caveolin-1: a critical regulator of lung injury. Am J Physiol Lung Cell Mol Physiol. 2011;300:L151-60.

30. Kunzmann S, Collins JJ, Yang Y, Uhlig S, Kallapur SG, Speer CP, et al. Antenatal inflammation reduces expression of caveolin-1 and influences multiple signaling pathways in preterm fetal lungs. Am J Respir Cell Mol Biol. 2011:45:969-76.

31. Gao C, Li R, Huan J, Li W. Caveolin-1 siRNA increases the pulmonary microvascular and alveolar epithelial permeability in rats. J Trauma. 2011:70:210-9.

32. Mitchell LA, Ward C, Kwon M, Mitchell PO, Quintero DA, Nusrat A, et al. Junctional adhesion molecule a promotes epithelial tight junction assembly to augment lung barrier function. Am J Pathol. 2015;185:372-86.
33. Fernandez AL, Koval M, Fan X, Guidot DM. Chronic alcohol ingestion alters claudin expression in the alveolar epithelium of rats. Alcohol. 2007:41:371-9.

34. Turi GJ, Ellis R, Wattie JN, Labiris NR, Inman MD. The effects of inhaled house dust mite on airway barrier function and sensitivity to inhaled methacholine in mice. Am J Physiol Lung Cell Mol Physiol. 2011;300:L185-90.

35. Daugherty BL, Mateescu M, Patel AS, Wade K, Kimura S, Gonzales LW, Guttentag S, Ballard PL, Koval M. Developmental regulation of claudin localization by fetal alveolar epithelial cells. Am J Physiol Lung Cell Mol Physiol. 2004;287:L1266-73.

36. Benediktsdóttir BE, Gudjónsson T, Baldursson Ó, Másson M. N-alkylation of highly quaternized chitosan derivatives affects the paracellular permeation enhancement in bronchial epithelia in vitro. Eur J Pharm Biopharm. 2014; 86(1):55-63.

37. Bhandari V. Hyperoxia-derived lung damage in preterm infants. Semin Fetal Neonatal Med. 2010;15:223-9.

38. Zhang QY, Fu JH, Xue XD. Expression and function of aquaporin-1 in hyperoxia-exposed alveolar epithelial type II cells. Exp Ther Med. 2014;8:493-8.

39. Babu PB, Chidekel A, Shaffer TH. Hyperoxia-induced changes in human airway epithelial cells: the protective effect of perflubron. Pediatr Crit Care Med. 2005:6:188-94.

40. Boueiz A, Hassoun PM. Regulation of endothelial barrier function by reactive oxygen and nitrogen species. Microvasc Res. 2009;77:26-34.

41. Cordes N, Hehlgans S, Eke I. Adhesion, invasion, integrins, and beyond. In The impact of tumor biology on cancer treatment and multidisciplinary strategies. Berlin: Springer-Verlag; 2009. p. 93-115

42. Gosens R, Mutawe M, Martin S, Basu S, Bos ST, Tran T, et al. Caveolae and caveolins in the respiratory system. Curr Mol Med. 2008;8:741-53.

43. Nag S, Venugopalan R, Stewart DJ. Increased caveolin-1 expression precedes decreased expression of occludin and claudin-5 during bloodbrain barrier breakdown. Acta Neuropathol. 2007;114:459-69.

44. Brott DA, Richardson RJ, Louden CS. Evidence for the nitric oxide pathway as a potential mode of action in fenoldopam-induced vascular injury. Toxicol Pathol. 2012:40:874-86.

45. Li S, Couet J, Lisanti MP. Src tyrosine kinases, Galpha subunits, and H-Ras share a common membrane-anchored scaffolding protein, caveolin. Caveolin binding negatively regulates the auto-activation of Src tyrosine kinases. J Biol Chem. 1996:271:29182-90.

46. Hardyman MA, Wilkinson E, Martin E, Jayasekera NP, Blume C, Swindle EJ, et al. TNF-alpha-mediated bronchial barrier disruption and regulation by srcfamily kinase activation. J Allergy Clin Immunol. 2013;132:665-75. e8.

47. Haines P, Samuel GH, Cohen H, Trojanowska M, Bujor AM. Caveolin-1 is a negative regulator of MMP-1 gene expression in human dermal fibroblasts via inhibition of Erk1/2/Ets1 signaling pathway. J Dermatol Sci. 2011;64:210-6.

48. Kim HN, Chung HS. Caveolin-1 inhibits membrane-type 1 matrix metalloproteinase activity. BMB Rep. 2008;41:858-62

49. Eum SY, Jaraki D, Bertrand L, Andras IE, Toborek M. Disruption of epithelial barrier by quorum-sensing $\mathrm{N}$-3-(oxododecanoyl)-homoserine lactone is mediated by matrix metalloproteinases. Am J Physiol Gastrointest Liver Physiol. 2014;306:G992-g1001.

50. Cao L, Wang H, Wang F. Amyloid-beta-induced matrix metalloproteinase-9 secretion is associated with retinal pigment epithelial barrier disruption. Int J Mol Med. 2013;31:1105-12.

51. Bucci M, Gratton JP, Rudic RD, Acevedo L, Roviezzo F, Cirino G, et al. In vivo delivery of the caveolin-1 scaffolding domain inhibits nitric oxide synthesis and reduces inflammation. Nat Med. 2000:6:1362-7.

52. Gratton JP, Lin MI, Yu J, Weiss ED, Jiang ZL, Fairchild TA, et al. Selective inhibition of tumor microvascular permeability by cavtratin blocks tumor progression in mice. Cancer Cell. 2003;4:31-9.

53. Zhu L, Schwegler-Berry D, Castranova V, He P. Internalization of caveolin-1 scaffolding domain facilitated by Antennapedia homeodomain attenuates PAF-induced increase in microvessel permeability. Am J Physiol Heart Circ Physiol. 2004;286:H195-201. 